Experimental Child Psychology

Elsevier Editorial System(tm) for Journal of

Manuscript Draft

Manuscript Number: JECP-D-15-00168R4

Title: Semantic processing of actions at 9 months is linked to language proficiency at 9 and 18 months

Article Type: SI:Language and Cognition

Keywords: action understanding; semantic processing; N400; language acquisition

Corresponding Author: Ms. Katharina Kaduk,

Corresponding Author's Institution:

First Author: Katharina Kaduk

Order of Authors: Katharina Kaduk; Marta Bakker; Joshua Juvrud; Gustaf Gredebäck, Professor; Gert Westermann, Professor; Judith Lunn, Dr; Vincent Reid, Dr

Abstract: The present study utilizes event-related potential methodologies to investigate how social-cognitive processes in preverbal infants relate to language performance. We assessed 9-month-old infants' understanding of the semantic structure of actions via an N400 ERP response to action sequences that contained expected and unexpected outcomes. At 9 and 18 months, infants' language abilities were measured using the Swedish Early Communicative Development Inventory (SECDI). Here we show that 9-month-old infants' understanding of the semantic structure of actions, evidenced in an N400 ERP response to action sequences with unexpected outcomes, are related to language comprehension scores at 9 months and are related to language production scores at 18 months of age. Infants who showed a selective N400 response to unexpected action outcomes are those who are classed as above mean in their language proficiency. The results provide evidence that language performance is related to the ability to detect and interpret human actions at 9 months of age. This study suggests that some basic cognitive mechanisms are involved in the processing of sequential events that are shared between two conceptually different cognitive domains and that pre-linguistic social understanding skills and language proficiency are linked to one another. 
Dear Dr. Gampe, Prof. Dr. Mani \& Prof. Dr. Daum,

Attached, please find our revised manuscript (Reference number: JECP-D-15-00168) with the title "Semantic processing of actions at 9 months is linked to language proficiency at 9 and 18 months", which we wish to resubmit to the Journal of Experimental Child Psychology Special issue. We would like to thank the reviewers for their latest comments and suggestions. We believe that we have been able to address all remaining minor comments to the reviewers' and editors' satisfaction.

Yours sincerely,

Katharina Kaduk, Marta Bakker, Joshua Juvrud, Gustaf Gredebäck, Gert Westermann, Judith Lunn and Vincent Reid. 


\section{Reviewer \#1:}

1.1. Thank you for attempting to provide the missing filter information. However, I wasn't asking about the online filter - the information is confusing and doesn't make sense. Please remove the following sentences: "This software filter is of Butterworth type, which is towards zero in the stopband in the passband with roll off. The Net Amps 300 use delta-sigma ADC technology with a digital antialiasing filter operating at $4 \mathrm{KHz}$. This provides a fixed hardware antialiasing filter with a frequency of 6 KHz."

- We have now removed the sentence from the manuscript as requested by reviewer 1 .

1.2 Instead, please add more information about the digital filter. On page 12, line 1 please revise the following sentence as following: "Subsequent data editing included digital filtering (0.3-30 Hz bandpass, [ADD YOUR INFORMATION HERE])..." The sentence could read like this: "Subsequent data editing included digital filtering (0.3-30 Hz bandpass, 3rd order Butterworth filter, forward and reverse)..."

We now include the filter type in the existing manuscript. The

- sentence on page 11 now states "... digital filtering $(0.3-30 \mathrm{~Hz}$ bandpass, Butterworth Finite Impulse Response filter, Passband Gain 99.0\% -0.1dB, Stopband Gain 1.0\% -40.0dB, filter roll-off 0.29Hz)..”

1.3. Also, I've tried to find with which analysis software the EEG analyses were done but couldn't find it in the manuscript. I might have missed it, but if not please also add this information.

- the data was post processed using Net Station. We have now included the information in the text on page 11 we state "Subsequent data editing was performed using Net Station 4.5.4 Waveform Tools and included digital filtering ..."

\section{Reviewer \#2:}

Thank you again for your thorough revisions. I believe two of my previous comments were misunderstood, so I clarify these below:

2.1. In response to the note about the other time windows, I was confused because the authors indicated that none of the comparisons were significant, but then all reported analyses listed $\mathrm{p}<.05$. My comment in the last revision assumed they were significant given the reported statistics. Were these all meant to be $p>.05$ ?:

- We apologize for the confusion. We would like to

confirm that all additionally reported statistics are $\mathrm{p}>.05$.

2.2. Although I appreciate that a difference between groups (main effect) is evident in Figure 4, I am still uncertain how this reflects the interaction with time. From my understanding, the time $\mathrm{X}$ condition interaction suggests that there is a peak in the expected case but not the unexpected case (Fig. 3). How is this peak defined and/or where can it be seen in Fig. 4 ? 
- We now provide guidance in the Figure 4 caption for the reader to understand the time $\mathrm{X}$ condition interaction. We have presented the data in this manner as we show the ERP morphology in the prior figure, yet there is no indication of the topographical aspects of this activity. Figure 4 facilitates a fuller understanding of the characteristics of the effects that we are reporting. The Figure 4 caption now states: "Difference wave (expected - unexpected) topographical maps for high \& low language comprehension groups at 9 months (top two) and high \& low language production group 18 months (bottom two) displaying every $50 \mathrm{~ms}$ of the 200 ms time window of interest. The effect is clearly seen in high language posterior bilateral regions in the highlighted area with a high intensity increase in microvoltage for the high language group from near the outset of the epoch, which continues for the duration of the epoch. In contrast, the low language group remains static and does not change in topography in terms of differential microvoltage for the entire epoch in the area of interest." 


\title{
Semantic processing of actions at 9 months is linked to language proficiency at 9 and 18 months
}

\author{
Katharina Kaduk ${ }^{\mathrm{a} 1}$, Marta Bakker ${ }^{\mathrm{b}}$, Joshua Juvrud ${ }^{\mathrm{b}}$, Gustaf Gredebäck ${ }^{\mathrm{b}}$, Gert \\ Westermann $^{\mathrm{a}}$, Judith Lunn ${ }^{\mathrm{a}}$, Vincent M. Reid ${ }^{\mathrm{a}}$ \\ ${ }^{a}$ Department of Psychology, Lancaster University, LAI 4YF, Lancaster, UK \\ ${ }^{b}$ Department of Psychology, Uppsala Child and Babylab, Box 1225, 751 42, Uppsala, \\ Sweden \\ ${ }^{1}$ Corresponding author at: Department of Psychology, Lancaster University, LA1 4YF, \\ Lancaster, UK \\ E-mail address: k.kaduk@lancaster.ac.uk (K. Kaduk).
}

\section{Acknowledgments}

This work was supported by FP7 Marie Curie ITN "ACT", Grant Number 289404. VR is a Reader and GW is a Professor in the International Centre for Language and Communicative Development (LuCiD) at Lancaster University. The support of the Economic and Social Research Council [ES/L008955/1] is gratefully acknowledged. We would like to express our gratitude to all of the families who participated in this research. The authors declare no competing interests. 
Semantic processing of actions at 9 months is linked to language proficiency at 9 and 18 months.

\begin{abstract}
The present study utilizes event-related potential methodologies to investigate how social-cognitive processes in preverbal infants relate to language performance. We assessed 9-month-old infants' understanding of the semantic structure of actions via an N400 ERP response to action sequences that contained expected and unexpected outcomes. At 9 and 18 months, infants' language abilities were measured using the Swedish Early Communicative Development Inventory (SECDI). Here we show that 9-month-old infants' understanding of the semantic structure of actions, evidenced in an N400 ERP response to action sequences with unexpected outcomes, are related to language comprehension scores at 9 months and are related to language production scores at 18 months of age. Infants who showed a selective N400 response to unexpected action outcomes are those who are classed as above mean in their language proficiency. The results provide evidence that language performance is related to the ability to detect and interpret human actions at 9 months of age. This study suggests that some basic cognitive mechanisms are involved in the processing of sequential events that are shared between two conceptually different cognitive domains and that pre-linguistic social understanding skills and language proficiency are linked to one another.
\end{abstract}




\section{Introduction}

Young infants show sophisticated abilities across an array of cognitive domains in early development (Mandler, 2006). One example of this is the ability to determine outcomes of actions, which is linked to the capacity to process other people's goals and their intentions (Baldwin et al., 2001). It is possible to conceptually divide human action into two primary forms. On the one hand, actions can be communicative in nature and are designed to directly engender social understanding of information. Within this framework, infants' preverbal social-communicative capacities such as the ability to follow others' eye gaze (e.g. Brooks \& Meltzoff, 2005, 2008, 2015), and to process pointing (e.g. Brooks \& Meltzoff, 2008; Butterworth \& Morissette, 1996; Tomasello, Carpenter, \& Liszkowski, 2007) and gestures (e.g. Kraljević, Cepanec, \& Šimleša, 2014; Rowe \& Goldin-Meadow, 2009a; 2009b) are well studied and have all been related to various aspects of later language abilities.

A second form of actions incorporates all those actions that are noncommunicative in nature but still convey goal directedness. Such goal directed actions can be observed repeatedly in typical environments by infants during early development, ranging from parents cleaning the home, to the preparation and consumption of food. In such scenarios, adults as well as young children readily construct action representations that are organized with respect to this ultimate goal (Baldwin, Baird, Saylor, \& Clark, 2001; Zacks et al., 2001). For example, infants from 6 months of age can accurately predict that a cup (Hunnius \& Bekkering, 2010) or a spoon (Kochukhova \& Gredebäck, 2010; Reid et al., 2009) should go towards the mouth rather than towards a person's ear. These representations allow for the prediction of the consequences of actions, including the ability to interpret and describe actions, and categorize action sequences (Sommerville \& Woodward, 2005). 
Prior research has shown that the structure of actions parallels that of linguistic utterances and that both actions and language show comparable hierarchical structures (Baldwin et al., 2001; Zacks et al., 2001). It has been argued that human language emerged from the hierarchical structure of instrumental actions. The same neural circuits that control the hierarchy of these instrumental actions served as a basis upon which the newly acquired function of language syntax has emerged (Gallese, 2007). The parallels that can be seen between the semantic organization of noncommunicative actions and the semantics within linguistic structures raise the possibility that processing of this action type in early development may be related to language. The context that is present within the execution of sentences and actions conveys information that facilitates the prediction of future events. Situational knowledge thereby provides the infant with a mechanism to use semantic rules during action observation (Ni Choisdealbha \& Reid, 2014). Amongst other cognitive and social advantages, this enables the infant to re-enact the final goal of a modelled action (Gergely, Bekkering, \& Kiraly, 2002) and to infer goals of an uncompleted action without seeing the achievement of the goal itself (Daum, Prinz, \& Aschersleben, 2008). Given these parallels in the organization of non-communicative actions and the structure of language, it has been conjectured that the ability to process these hierarchically structured actions in early development may pave the way for language acquisition (Reid et al., 2009). From the evolutionary account, it has been suggested that language has its origin in the ability to interpret others' gestures and actions (e.g. Corballis, 2003; Rizzolatti \& Arbib, 1998). Together, these findings indicate that in addition to previous findings, which demonstrated strong relations between infants' preverbal social-communicative capacities and language and have already been well studied, understanding of non-communicative action may also be 
linked to the development of language or share similar domain-general processes. To date, there is no empirical evidence to support the notion that links between the two domains exist in early development. Consequently, the present study investigates the relation between the semantic processing of non-communicative, instrumental actions during infancy, that is the ability to detect and interpret other's action end states as either expected or unexpected, and relate this ability to language abilities in the first and second postnatal year.

In language research, the N400 component of the event-related potential (ERP) has been identified as a neural signature related to the formation of a semantic representation, as the $\mathrm{N} 400$ is elicited when a word does not fit an expected context (e.g. Kutas \& Hillyard, 1980). In the action domain, N400 effects are observable when action outcomes are violated in infants and adults (e.g. Parise \& Csibra, 2012; Reid \& Striano, 2008; Reid et al., 2009; Wu \& Coulson, 2005). In adults the N400 has also been found to be sensitive to the relation between gesture and speech (Holle \& Gunter, 2007; Kelly et al., 2007). The similarity in the electrophysiological responses to semantic violations in the action and language domain suggests that language processing may derive from understanding action in early development, or to some extent, share similar cognitive mechanisms. Should infants first start to understand the parameters of semantic structures within the action domain, this capacity may well bootstrap learning about language. Pre-linguistic semantic processing of action sequences contain hierarchically aligned structures that are similar to that of sentential structures. As such, the capacity to semantically process action may therefore be related to language capacities.

A key question, that has to date remained unanswered, is whether language capacities are based on structures initially detected and interpreted within the action 
domain. For this purpose we presented 28 nine-month-old infants with a sequence of images with expected and unexpected action outcomes known to reliably induce an N400-like ERP component over parietal regions (Reid et al., 2009). In addition to the N400 effect, Reid et al. (2009) also found a Negative central (Nc) component, which was larger in amplitude for the expected condition over frontal and central areas when contrasted with the unexpected condition. The $\mathrm{Nc}$ is thought to reflect attentional processes with greater amplitude for stimuli that elicit a higher allocation of attentional resources (Reynolds \& Richards, 2005) and is typically found over frontal and central sites, peaking at around 300 to $700 \mathrm{~ms}$ after stimulus onset (Webb, Long, \& Nelson, 2005). Previously, the Nc has been found to be more enhanced for familiar stimuli, for instance familiar versus novel faces (e.g. deHaan \& Nelson, 1997). Reid et al. (2009) argued that their observed Nc effect was driven by infants' higher interest in stimuli depicting food consumption. An alternative and equally plausible explanation given by the authors could be that 9-month-olds are capable of judging where food should be placed when people hold and direct food towards the head area, as these are actions that infants are more likely to be exposed to in everyday life. Therefore, a familiarity effect for the expected action conclusion could drive the observed Nc effect. Our study employs the same stimuli and age group as Reid et al., (2009). We therefore expected to replicate the morphology of the Nc and N400 in Reid et al. (2009) within the present study.

In order to investigate expected and unexpected goal directed action processing at 9 months and infants' language abilities, we assessed language skills at 9 months as well as when the same children reached the age of 18 months by introducing two forms of the Swedish Early Communicative Development Inventory (SECDI, Eriksson, Westerlund, \& Berglund, 2002). In order to assess infants' action 
processing abilities and relate these to language proficiency, percentile scores from both the SECDI words and gestures (w\&g) at 9 months and SECDI words and sentences (w\&s) at 18 months were used to split our sample at the $50^{\text {th }}$ percentile (for a similar paradigm see Torkildsen et al., 2009). Consequently we examined infants' semantic action processing abilities at 9 months (via obtained ERP data) separately for each of the two language proficiency groups and time points (here termed low and high language comprehension at 9 months \& low and high language production at 18 months). We hypothesized that if the application of rules relating to the structure of action were also used when processing language structures, then only the group of infants with an above mean performance in language comprehension and production at both time points of the language assessment will display evidence for semantic processing within the action domain, as indexed by the N400 ERP component.

\section{Method}

\section{Participants}

The final sample comprised 28 nine-month-old monolingual Swedish infants (14 female) with a mean age of 277 days (range 262-287 days). When split at the $50^{\text {th }}$ percentile on the SECDI at 18 months, the low language production group $(n=14,6$ female) had an age range at 9 months between 263-284 days $(M=272)$ and the high language production group ( $n=14,8$ female) had an age range between 262-287 days $(M=276)$. The average age during the follow up language production assessment at age 18 months for the low language group was 556 days (range: 540-573), and for the high language group 557 days (range: 547-573). An additional 25 infants were tested but excluded due to inattentiveness, therefore providing fewer than the required minimum number of 5 artifact-free trials per condition for data analysis. A meta- 
analysis on 149 published infant EEG studies demonstrated an average attrition rate of $49.2 \%$ (Stets, Stahl, \& Reid; 2012). Thus, an attrition rate of $47.2 \%$ as present in this study is within norms in this research field. Eleven remaining infants were excluded due to technical failure $(n=6)$, or parents failing to complete the follow up language questionnaire $(n=5)$. All infants were born full term $(37-42$ weeks $)$ and were of normal birth weight (> 2500g). Each participant's parent gave informed written consent and received a gift voucher for participation. This research was approved by the local ethical committee, which stands in accordance with the 1964 Declaration of Helsinki.

\section{Materials}

Experimental stimuli were the same as those used by Reid et al., (2009). They were composed of three photographs depicting a male or a female actor, showing eating actions either by holding a spoon, a fork or the food itself. The sequence of the action followed a hierarchical order always first depicting (A) the action context, (B) mid action, and action conclusion that either (C) conformed or (D) violated the expected action outcome (Fig. 1).

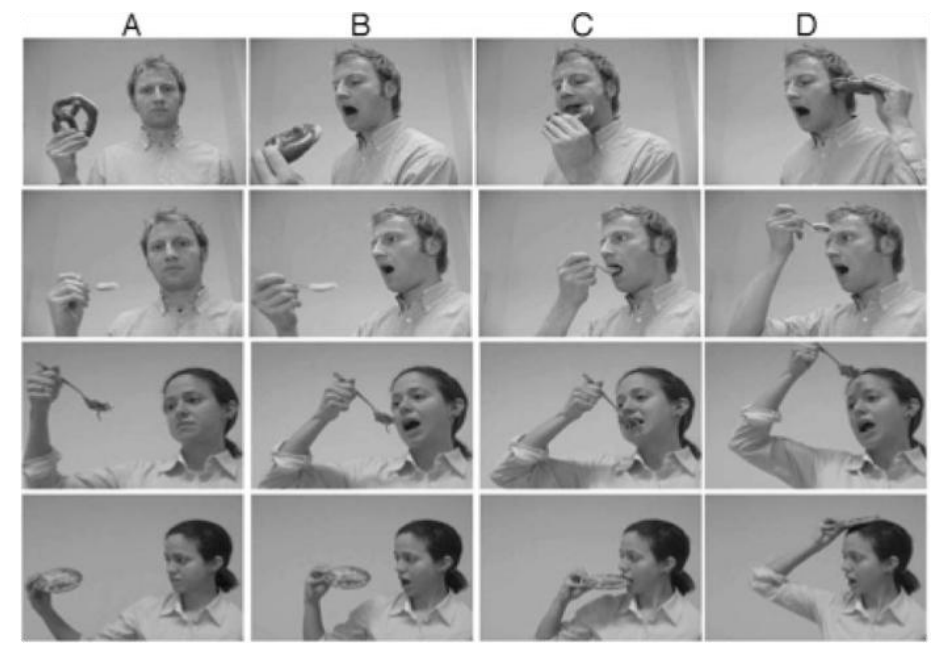

Fig. 1. ERP stimuli: 3-image sequence depicting (A) context, (B) mid action and action outcome (C- 
D). The N400 and Nc components were assessed for the last image of the sequence depicting the expected (C) or unexpected (D) action outcome.

Stimuli were shown on a 17 -inch computer monitor $(37 \times 31 \mathrm{~cm})$ at a viewing distance of $90 \mathrm{~cm}$ via E-Prime 2.0, E-Studio (Psychology Software Tools, Inc., Pittsburgh, PA) and were $22 \times 17.5 \mathrm{~cm}$ in size. This produced a visual angle of 15.2 degrees (horizontal) and 12.7 degrees (vertical). Images A and B were presented for the duration of $750 \mathrm{~ms}$ and C-D for $1000 \mathrm{~ms}$ and all three images were presented consecutively. The eight possible 3-image sequences were presented at random with the constraints that neither the same condition (congruent or incongruent), nor the same actor (male or female), were presented more than 2 times consecutively. The stimuli described in the present study were intermixed with other visual stimuli not relevant to this study (a hand displaying a give-me gesture and a control hand configuration). Both stimulus sets were presented in separate blocks. Each block varied in length ranging from 3 to 8 presentations. The two blocks were presented one after the other throughout the experimental session to ensure an equal frequency distribution and the starting order was counterbalanced between subjects. To eliminate expectancy effects the ISI between blocks, as well as between each 3-image sequence and the stimuli from the second block, were always presented for a random duration of 300 to $600 \mathrm{~ms}$ and in the center of the monitor on a grey background. Each stimulus and block was followed by a black fixation cross on the same grey background, which again lasted for a random duration of 300 to $600 \mathrm{~ms}$. The N400 and $\mathrm{Nc}$ components were assessed for the last image of the 3-image sequence depicting the (expected or unexpected) action outcome.

Language measure: The SECDI (Eriksson, Westerlund, \& Berglund, 2002) is the 
Swedish adaptation of the MacArthur Communicative Development Inventory (Fenson et al., 1993; 1994), measuring communicative comprehension and production skills in young children based on parental reports. Two versions are available and are designed for the use of different age groups. The SECDI- words and gestures (w\&g) is preliminary designed to assess language comprehension of children aged 8 to 16 months and the SECDI words and sentences (w\&s) is designed for children aged 16 to 28 months, which only measures language production. We used the SECDI w\&g at the first testing point at 9 months and the latter to investigate infants' language production abilities at 18 months. The SECDI w\&g is normed on 228 and the SECDI w\&s is normed on 336 Swedish children and further provides monthly norms by means of interpolation from existing data (see Berglund \& Eriksson, 2000).

\section{Procedure}

During the ERP session at 9 months of age, infants were seated on the caregiver's lap in a dimly lit experimental room. The experimenter was present in the same room but separated from the parent and infant by a curtain, thereby allowing the experimenter to monitor each infant's behavior via a control monitor. This enabled the experimenter to pause the experiment and offer breaks each time the infant became inattentive or fussy. For the duration of the pause, the monitor remained blank. The experiment ended once the infant's attention could no longer be maintained, but did not exceed 10 minutes in total. The infant was video-recorded throughout the session for offline coding of looking behavior to the experimental stimuli and to control for the amount of motor activity in each trial. After the ERP session caregivers received the SECDI w\&g together with a prepaid envelope and were asked to fill out and return the language questionnaire within one week of their first visit per post. 
Participants were followed up 9 months later by contacting the caregivers a week before the infant was due to become 18 months of age. Caregivers were asked to fill out the SECDI w\&s online via a link that was provided by the experimenter. Percentile scores from the SECDI w\&g and w\&s (Berglund \& Eriksson, 2000) language measures at 9 and 18 months were used to assign infants to one of two groups, namely low language and high language. In line with previous research (e.g. Torkildsen et al., 2009) we defined the border that separated the low from the high language group at the $50^{\text {th }}$ percentile. According to the SECDI manual (Eriksson, Westerlund, \& Berglund, 2002), the average number of words comprehended by Swedish 9-month-olds is 11 words. At age 18 months the reported word production reaches 35 words on average. Children in the high language comprehension group at 9 months understood a mean of 34.5 words (range: 15-102) and children in the low language comprehension group at 9 months had a mean total comprehensive vocabulary of 5.6 words (range: 0-11). When followed up, children in the high language production group at 18 months had a mean of 163.4 words (range: 42-484) and the low language production group had a mean total productive vocabulary of 20.4 words (range: 6-33). An independent samples t-test confirmed that language scores were significantly higher for infants from the high language comprehension group at 9 months $(M=74.0, S D=14.0)$ than the low language comprehension group at 9 months $(M=29.2, S D=15.1), t(25)=7.99, p<.001, d=3.08$, as well as for the high language production group at 18 months $(M=82.9, S D=9.9)$ in comparison to the low language production group at 18 months $(M=34.3, S D=$ $14.5), t(26)=10.3, p<.001, d=3.9$, thereby justifying the split at the $50^{\text {th }}$ percentile.

However, the two obtained language measures (9 months comprehension and 18 months production vocabulary) did not correlate with one another $r(26)=.21, p=$ 
0.19. This is due to the fact that only half the sample ( $n=14,8$ high language group, 6 low language group) remained within the same language proficiency group for both time points. Six children moved up (from low language comprehension at 9 months to high language production at 18 months) and 7 children moved down in their language proficiency (from high comprehension at 9 months to low production at 18 months). From one child we were only able to obtain language scores for the second time point. Consequently, this child was excluded from any analysis concerning 9 months language proficiency comparisons. Thus, children were as equally likely to remain, move up or downward in their language proficiency.

It should be noted that word production is preferentially used to mark toddlers language skills, as productive vocabulary is likely to be a more reliable indicator when relying on parental reports to determine language proficiency (Rämä, Sirri, \& Serres, 2013). Language production is therefore more commonly used in studies investigating links between language and the N400 (e.g. Friedrich \& Friederici, 2006, 2010; Mills et al., 2005; Torkildsen, 2006, 2008, 2009). Our a priori main focus was therefore to assess the relation between word production and the action N400.

\section{EEG recording and analysis}

EEG was acquired via 128-channel Geodesic Sensor Nets, amplified with an EGI Net Amps 300 amplifier with a sampling rate of 250Hz (HCGSN 130; EGI, Eugene, OR). EEG was further referenced online to the vertex electrode $(\mathrm{Cz})$ and an analogue hardware bandpass filter of $0.1-100 \mathrm{~Hz}$ was applied with data stored for off-line analysis. Subsequent data editing was performed using Net Station 4.5.4 Waveform Tools and included digital filtering $(0.3-30 \mathrm{~Hz}$ bandpass, Butterworth Finite Impulse Response filter, Passband Gain 99.0\% -0.1dB, Stopband Gain 1.0\% -40.0dB, filter 
roll-off $0.29 \mathrm{~Hz}$ ) and data segmentation $(200 \mathrm{~ms}$ prior and $1000 \mathrm{~ms}$ after stimulus onset). The $200 \mathrm{~ms}$ baseline used for segmentation represented the last $200 \mathrm{~ms}$ of the mid action (B) image (for the same procedure see Reid et al., 2009). Off-line inspection of the video recordings ensured that only trials in which infants paid attention to all three images of the given sequence were further processed. The remaining trials were manually and visually inspected for artifacts caused by eye or body movements utilizing normative procedures for infant ERP studies (see Hoehl \& Wahl, 2012, for further information on infant ERP methods). An artifact detection tool was applied and EEG data in affected channels was marked as bad whenever amplitude exceeded the set threshold of \pm 200 microvolts for the entire segment, with a moving average of $80 \mathrm{~ms}$. Trials including more than 10 channels with excessive noise levels were rejected. Noisy channels from the remaining trials, that comprised fewer than 10 bad channels, were interpolated by using the average of surrounding electrodes. Remaining artifact-free trials were re-referenced to the average electrode and baseline corrected before creating individual averages for each participant. The grand average was produced from those 28 individuals that contributed 5 trials to their average per condition. Because the second image also serves as the baseline for the third image, we did not allow for any shifts in attention throughout the 3 -image sequence, as this would have been reflected in a noisy baseline. On average, infants saw 34.2 (range: 20-48) 3-image sequences for the expected and 34.3 (range: 21-49) for the unexpected condition. The probability of attending to all three images of the sequence decreased with the length of the testing session. Based on findings from Stets and Reid (2011) as well as Kaduk, Elsner and Reid (2013), reliable and interpretable data has previously been shown to be present within infant EEG with as few as 3 to 7 trials per condition using standard visual ERP paradigms. The Nc 
component throughout the present analysis is therefore useful as a guide to indicate the morphological quality of the present dataset. A very commonly observed problem, besides infant's general lack of attention in ERP studies, is neural habituation. Neural habituation potentially alters the outcome of obtained ERP morphology. Stets and Reid (2011; see also e.g. Snyder, Webb, \& Nelson, 2002) have reported that infants habituation to stimuli causes a shift of attention over the course of an experimental session. The inclusion of trials obtained nearer the end of an acquisition session can attenuate earlier effects with the potential to even produce opposite effects. Infants from the present study largely differed in the amount of trials they were able to contribute to their individual averages between subjects, ranging between 5 and 20 trials for the expected and 5 and 21 for the unexpected condition. Including "as many trials as possible" would potentially mask any Nc effect, if taking into account the changes in amplitude through neural habituation. Therefore, rather than including as many artifact-free trials for each participant's individual average and condition as possible, we decided to analyze only the first 5 artifact-free trials. Furthermore, the inclusion of a larger sample of infants compensates for the low inclusion criterion. As there is currently no evidence that a larger number of trials improves the morphology of the N400 component in early development, we felt that by replicating the Nc effect found in Reid et al. (2009), we will further provide evidence for the validity of this approach and would justify the employment of this technique with the N400.

\section{Results}

Nc ERP Component

Visual inspection of the Nc effect led to the identification of 10 channels representing the left central scalp $(29,30-\mathrm{C} 1,35,36-\mathrm{C} 3,37,40,41-\mathrm{C} 5,42,46,47)$ 
and 10 channels representing the right central scalp (87, 93, 98, 102, 103-C6, 104-C4, $105-\mathrm{C} 2,109,110,111)$, which were in line with topographical locations for the $\mathrm{Nc}$ present in prior infant research (e.g., Parise, Reid, Stets, \& Striano, 2008). A time window was chosen from 350 to $600 \mathrm{~ms}$ post stimulus presentation in order to capture the primary peak of the component.

For the overall sample $(n=28)$ we first performed a $2 \times 2$ repeated measures ANOVA with the within subject factors condition (expected, unexpected) and hemisphere (left, right). Statistical analysis revealed a significant main effect for condition, $F(1,27)=4.37, p=.046$, partial $\eta^{2}=.139$.
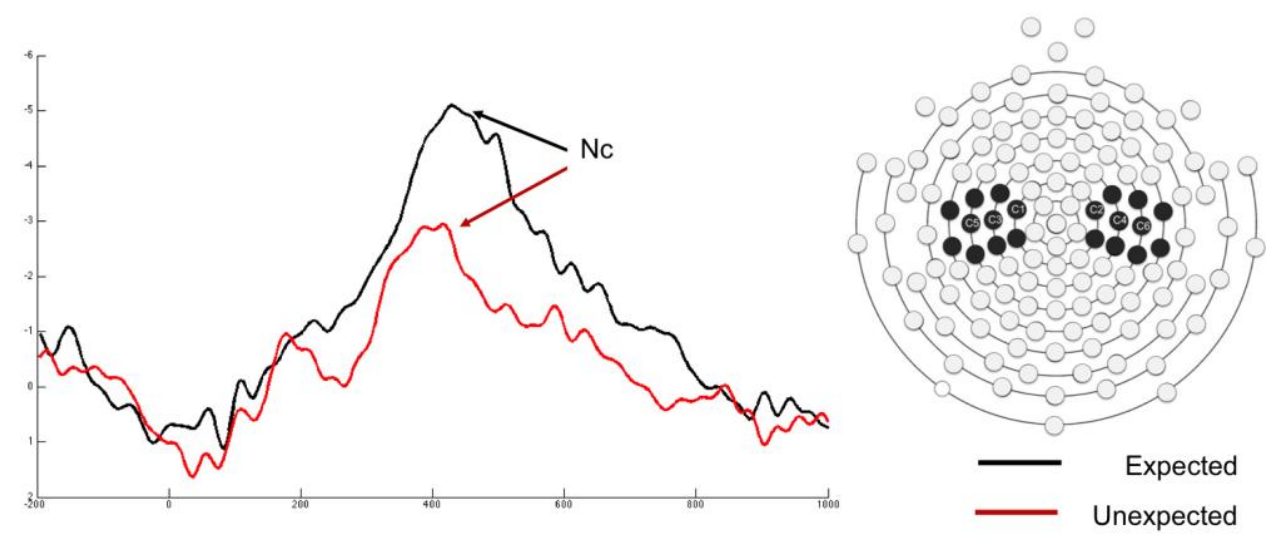

Fig. 2. Left : Averaged ERPs for all infants $(\mathrm{N}=28)$ from 20 channels, 10 of the left central $(29,30-\mathrm{C} 1$, $35,36-\mathrm{C} 3,37,40,41-\mathrm{C} 5,42,46,47)$ and 10 of the right central regions $(87,93,98,102,103-\mathrm{C} 6,104-$ C4, 105-C2, 109, 110, 111), displaying the Nc. Right: Analyzed area of interest with reference to the 10-20 system.

Nc: Language division from the SECDI $w \& g$ for language comprehension

When dividing the sample into groups according to their language comprehension abilities at 9 months of age, the $2 \times 2$ repeated measures ANOVA with the within subject factors condition (expected, unexpected) and hemisphere (left, 
right) and the between subject factor language comprehension revealed a significant main effect for condition, $F(1,26)=4.45, p=.045$, partial $\eta^{2}=.151\left(M_{\text {expected }}=-3.83\right.$, $\left.S D=4.05, M_{\text {unexpected }}=-1.93, S D=3.51\right)$. No other main effects or interactions were found (all $\mathrm{p}$ values $>.05$ ).

\section{Nc: Language division from the SECDI $w \&$ s for language production}

For the groups split according to the SECDI w\&s at 18 months, via which language production was measured, the 2 (condition) $\times 2$ (hemisphere) repeated measures ANOVA with the between subject factor language production revealed a significant main effect for condition, $F(1,27)=4.35, p=.047$, partial $\eta^{2}=.143$ $\left(M_{\text {expected }}=-3.62, S D=4.13, M_{\text {unexpected }}=-1.79, S D=3.53\right)$. No other main effects or interactions were found (all p values $>.05$ ).

A follow up paired sample t-test comparing both groups (high \& low producers) for the central electrodes confirmed these results as no significant differences between conditions were found in the high language production group, $t(13)=-1.985, p=.069, d=.69\left(M_{\text {expected }}=-3.72, S D=3.61, M_{\text {unexpected }}=-1.07, S D=\right.$ 4.08) or with the low language producers, $t(13)=-.881, p=.394, d=.26\left(M_{\text {expected }}=-\right.$ $\left.3.51, S D=4.7, M_{\text {unexpected }}=-2.51, S D=2.85\right)$.

\section{N400 ERP Component}

For the analysis of the N400, a time window around 600 to $800 \mathrm{~ms}$ after stimulus onset in parietal sites was chosen based on prior literature (Reid, et al., 2009) and the observed ERP morphology, as the current research is the first to report on N400 to action with this population utilizing a high density EEG array. Based on visual inspection of the individual and grand averages, we chose 16 electrodes to 
represent the parietal region $(52-\mathrm{P} 3,53,58,59,60,61,62-\mathrm{Pz}, 67,72,77,78,85,86$,
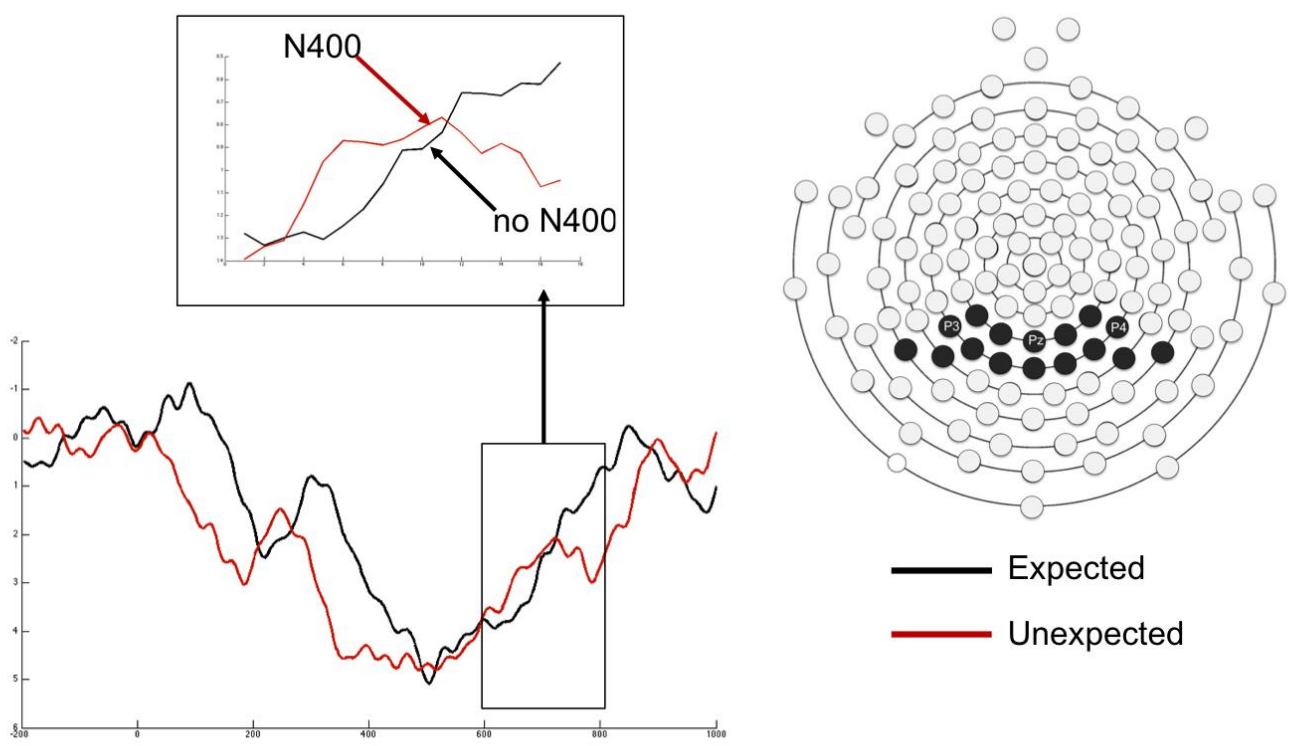

Fig. 3. Bottom left: Averaged ERPs for all infants $(\mathrm{N}=28)$ from 16 channels within the parietal region for the N400 (52-P3, 53, 58, 59, 60, 61, 62-Pz, 67, 72, 77, 78, 85, 86, 91, 92-P4, 96). Top right: Zoomed in time window of interest indicating the N400 for the unexpected and no N400 for the expected condition. Right: Analyzed area of interest with reference to the 10-20 system.

The data was consequently analyzed utilizing a time window analysis technique (Hoorman, Falkenstein, Schwarzenau, \& Hohnsbein, 1998), which we performed overall as well as separately for each group (low and high language), to assess the morphology of the N400 component within each of the groups. Time window analysis is used when one condition displays a defined peak in the window of interest, whereas the other does not. Time is therefore included as an additional within subject factor in the repeated measures analyses of variance (ANOVA) analysis. A significant interaction between condition and time indicates validity for the 
hypothesis that the N400 component is only evident for the unexpected condition as a function of variance over time. The repeated measure ANOVA therefore featured the two factors condition (expected, unexpected) and time (17 samples at one sample per 12ms) - as suggested by Hoorman et al. (1998), and further applied by Reid et al. (2009) and Domínguez-Martínez, Parise, Strandvall and Reid (2015) in N400 research. We selected 17 samples at $12 \mathrm{~ms}$ as this best reflected the difference between conditions. First, to confirm previous findings from Reid et al. (2009), we examined significant interactions over the entire sample. Results for the overall sample confirmed the observed morphology of the N400 effect for the unexpected but not the expected condition, with a significant condition and time interaction (GreenhouseGeisser correction), $F(3.6,98.0)=3.273, p=.018$, partial $\eta^{2}=.108$.

\section{Analysis Considerations}

However, for the N400 time window analysis (Hoorman et al., 1998) a threeway interaction would be very difficult to establish when there is an unequal amount of variance across the two groups, which is what is predicted and shown from this type of analysis. The variance in the low language group would need to be considerably more homogenous for a three-way interaction to emerge. In cases like this, when looking directly at variability in developmental data, it is strongly recommended that groups be addressed separately (Thomas et al., 2009). We therefore hypothesized that we would find a significant interaction between condition and time exclusively for the high language production group when further dividing the two groups according to language proficiency.

N400: Language division from the SECDI $w \& g$ for language comprehension 
The results from the 2 (condition) $\mathrm{x} 2$ (time) ANOVA with the between subject factor 9-months comprehension showed a significant interaction between condition and time (Greenhouse-Geisser correction), $F(3.5,86.8)=3.043, p=.027$, partial $\eta^{2}=.109$. No 3-way interaction with the additional between subject factor language comprehension was evident. We further divided the sample according to language proficiency at 9 months of age. We again performed a 2 × 2 ANOVA and found a significant interaction between condition and time for the high language comprehension group at 9 months, $F(2.5,34.4)=3.386, p=.037$, partial $\eta^{2}=.195$. Infants from the low language comprehension group at the 9 months language split did not produce a significant effect for the condition and time interaction, $F(3.6,39.3)$ $=1.182, p=.332$, partial $\eta^{2}=.097$.

\section{N400: Language division from the SECDI $w \&$ s for language production}

Results from the 2 (condition) x 2 (time) ANOVA with the between subject factor 18-months production revealed a significant condition and time interaction, $F(3.6,92.63)=3.225, p=.020$, partial $\eta^{2}=.110$. The $2 \times 2$ ANOVA performed for the two language proficiency groups separately revealed a significant interaction only for the high language proficiency group when split according to the language measure assessed at age 18 months, $F(3.5,46.1)=4.075, p=.009$, partial $\eta^{2}=.239$. Infants from the low language production group did not produce a significant effect for the condition and time interaction, $F(2.9,37.4)=.901, p=.446$., partial $\eta^{2}=.065$. To illustrate the interaction over time we computed a difference wave topographical map (expected - unexpected) for all 4 language proficiency groups over the course of 200 $\mathrm{ms}$ in the observed time window of interest between 600 and $800 \mathrm{~ms}$ (Fig. 4). 


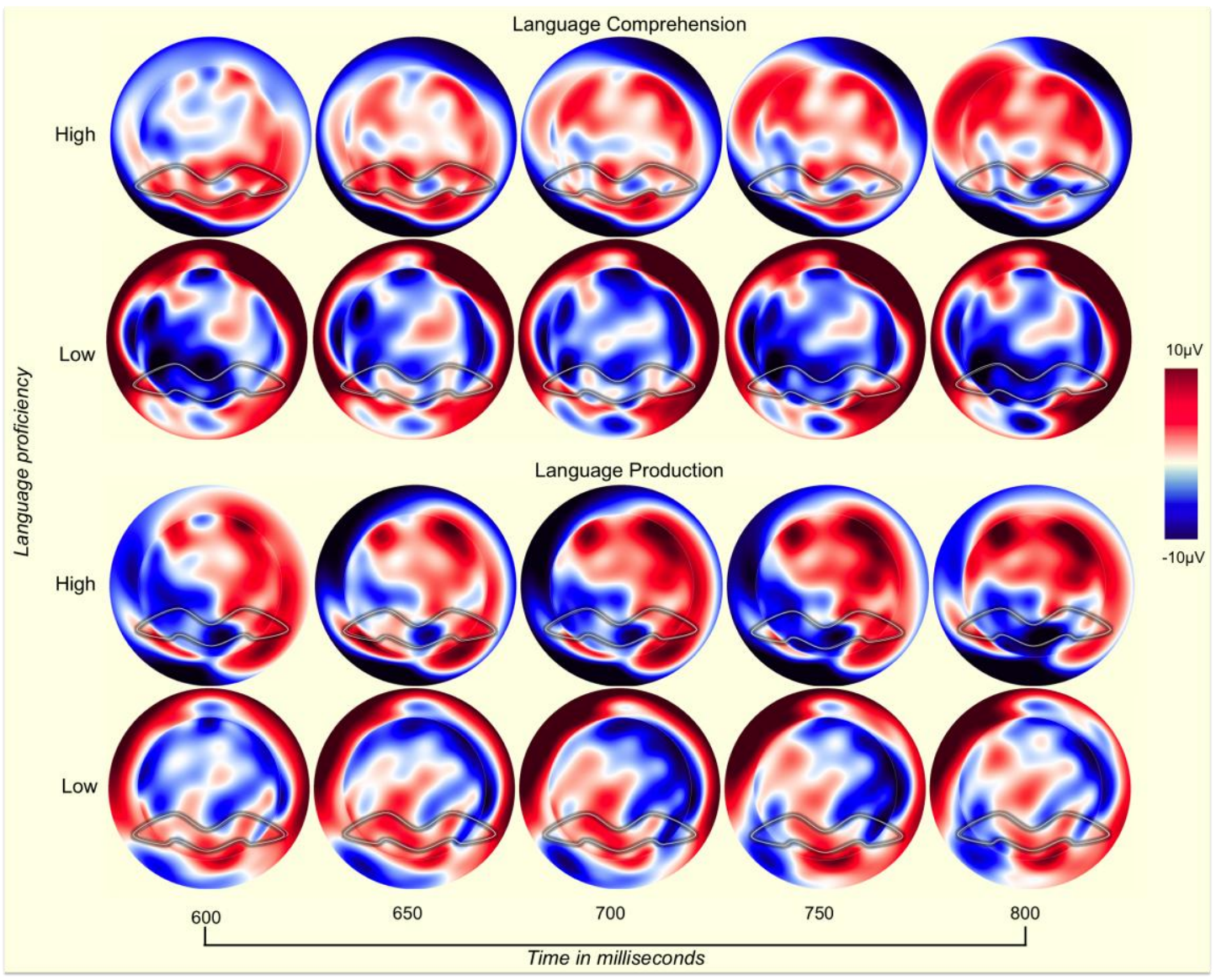

Fig.4. Difference wave (expected - unexpected) topographical maps for high \& low language comprehension groups at 9 months (top two) and high \& low language production group 18 months (bottom two) displaying every $50 \mathrm{~ms}$ of the $200 \mathrm{~ms}$ time window of interest. The effect is clearly seen in high language posterior bilateral regions in the highlighted area with a high intensity increase in microvoltage for the high language group from near the outset of the epoch, which continues for the duration of the epoch. In contrast, the low language group remains static and does not change in topography in terms of differential microvoltage for the entire epoch in the area of interest.

\section{Discussion}

The present study utilized event-related potential techniques to investigate how the understanding of non-communicative actions in early development may relate to language capacities later in life. Our results successfully replicated both the N400 and the Nc component in related regions of the scalp and time windows as those reported 
in Reid et al. (2009), with the ERPs following the same morphology despite the use of a high-density electrode array, whereas the original study had less than 30 channels for the overall sample.

When looking at the obtained ERP data by dividing the group as a function of language comprehension at 9-months, the N400 effect was only evident in the group of children who scored higher on the SECDI w\&g. The same pattern of effect remained stable when dividing our ERP results based on language production capacities at 18 months. Overall we can draw the conclusion that the ability to judge whether an observed non-communicative human action is to be expected or not on the semantic level is closely related to language performance. During the processing of expected and unexpected actions, only the high language production group appeared to differentiate the two actions as indexed by the presence of the N400 ERP component in the unexpected action condition.

The overall main effect for condition for the Nc component over the entire sample indicated that more attentional resources were used to process the expected, arguably more familiar, action outcomes. When the group was divided by language comprehension abilities at 9 months, as well as high and low production at 18 months, neither language group were able to discriminate between the familiar and unfamiliar action outcomes. The general conclusion that can be drawn from this is that both groups seem to be equally capable of detecting the familiar from the unfamiliar action outcomes by allocating more attention to the more familiar action outcomes.

We divided our sample at the 9-months comprehension level as well as the 18months language production level. Children in the present study were equally likely to remain within the same language proficiency group as they were to move up or down in their language proficiency. These results confirm the generally perceived weakness 
across literature towards the reliability of parental reports on the comprehension level (e.g. Friedrich \& Friederici, 2006, 2010; Mills et al., 2005; Rämä, Sirri, \& Serres, 2013; Torkildsen, 2006, 2008, 2009). It is therefore important to stress that any analyses reported in this study and interpretations that are based on splits at the 9months comprehension level have to be taken cautiously. Although we were not able to find 3-way interactions with language proficiency as the between subject factor in our performed repeated measures ANOVAs, the targeted statistical analyses reported in the current study mirrors prior research investigating the link between language proficiency and the language N400 (e.g. Friedrich \& Friederici, 2010). Due to our a priori hypothesis, separate analyses as a function of language capacity were undertaken.

Prior work has shown that aspects of social communication are linked to later language development. The observed effects of the present study occur earlier in ontogeny when compared with other known aspects of social understanding, including periods where the ability to interpret eye gaze (Brooks \& Meltzoff, 2005) or gestures (Butterworth \& Morissette, 1996; Rowe \& Goldin-Meadow, 2009a; 2009b; Tomasello et al., 2007) predict later language proficiency. The current study links with prior language development research but provides a different pathway in the attempt to understand the development of language. Specifically, the present study investigates non-communicative human actions using neurophysiological measures of action semantics reflected in the N400 ERP component. Here we suggest that language proficiency is aligned with semantic processing abilities in development. The current data suggests that these competencies in action may co-occur or bootstrap learning about language throughout development. When contrasted with previous work investigating early social skills and later language abilities (e.g. Brooks \& 
Meltzoff, 2005, 2008), the current study introduces an additional measure in the form literature could not comment on the underlying structure of cognitive processes as all prior literature is based on inference from behavioural results. The ERP approach provides a basis for a stronger conclusion in terms of the specific cognitive activities related to processing action sequences. Rather than indicating that relations exist between the action and language domain, the current study allows for the assessment of a specific cognitive mechanism in the form of the N400. As the N400 is only involved during semantic tasks, this cognitive process must be involved in the processing of actions. It is therefore possible to conclude that the semantic processes that are engaged during action observation are linked to language proficiency. With regards to the stability and predictability of language development, we need to stress that measures of language during infancy and early childhood are thought to have issues with reliability. Specifically, measures of language capacities are thought to become increasingly more consistent later in development (Bornstein \& Putnick, 2012). As such, our measures at 9 months of age should be taken with caution

Other aspects of action prediction not assessed in the current study may well be related to later language skills. For example, the relation between N400 action semantics and N400 language semantics are not yet explored. Further studies are required to map the parameters of these processes across development by using a more refined measure for language comprehension earlier in development.

This study assessed an electrophysiological component, where the participants required no overt behavioral response. The learning mechanisms underlying our findings may be explained via the Directed Attention Model (Reid \& Striano, 2007) that has implications for understanding social learning in infancy. In this model, 
different aspects of information lead to more refined processing of environmental variables. This allows the infant to focus limited attentional resources to optimize the outcome of any social interaction. For example, the early detection of semantic structures in actions and the enhanced attentional allocation to familiar action outcomes will allow the infant to disambiguate action which may help determine the referent of words or to understand sentences. The Nc effects reported in the present study align well with such an interpretation of the development of social processing skills during infancy, with attention allocated to specific action outcomes.

\section{Conclusions}

These results provide evidence for a developmental account that focuses on how language function emerges from pre-linguistic social understanding skills over the first postnatal year. Following these results, experiments can now be performed whereby the nature of this relation can be further examined, with the N400 as a primary tool to determine the associations between action and language processing in early development. This work highlights the parallels in the organization of actions and language, demonstrating that some basic cognitive mechanisms involved in the processing of sequential events are shared between conceptually different cognitive domains that already co-exist early in development. Further, this study raises the intriguing concept that understanding actions in early development may to some extent evolve at the same time as language acquisition or act as a mediator for linguistic skills. Finally, on the basis of these data, it is clear that observed overall effects may well provide important information related to development when examined via an individual differences approach. 


\section{References}

Baldwin, D. A., Baird, J. A., Saylor, M. M., \& Clark, M. A. (2001). Infants parse dynamic action. Child Development, 72, 708-717.

Berglund, E., \& Eriksson, M. (2000). Communicative development in Swedish children 16-28 months old. The Swedish early communicative development inventory - words and sentences. Scandinavian Journal of Psychology, 41, 133-144.

Bornstein, M. H. \& Putnick, D. L. (2012). Stability of language in childhood: A multiage, multidomain, multimeasure, and multisource study. Developmental Psychology, 48(2), 477-491.

Brooks, R. \& Meltzoff, A. N. (2005). The development of gaze following and its relation to language. Developmental Science, 8, 535-543.

Brooks, R. \& Meltzoff, A. N. (2008). Infant gaze following and pointing predict accelerated vocabulary growth two years of age: A longitudinal growth curve modeling study. Journal of Child Language, 35, 207-220.

Brooks, R. \& Meltzoff, A. N. (2015). Connecting the dots from infancy to childhood: A longitudinal study connecting gaze following, language, and explicit theory of mind. Journal of Experimental Child Psychology, 130, 67-78.

Butterworth, G. E., \& Morissette, P. (1996). Onset of pointing and the- acquisition of language in infancy. Journal of Reproductive Infant Psychology, 14, 219-231.

Corballis, M. C. (2003). From Hand to Mouth: The Gestural Origins of Language. In M. H. Christiansen, \& S. Kirby. (Eds.), Language Evolution (pp. 201-218). Oxford University Press, Inc., New York.

Daum, M., Prinz, W., \& Aschersleben, G. (2008). Encoding the goal of an object 
directed but uncompleted reaching action in 6- and 9-month-old infants. Developmental Science, 11, 607-619.

deHaan, M. \& Nelson, C. A. (1997). Recognition of the mother's face by six-month old infants: a neurobehavioral study. Child Development, 68, 187-210.

Domínguez-Martínez, E., Parise, E., Strandvall, T., \& Reid, V. M. (2015). The Fixation Distance to the Stimulus Influences ERP Quality: An EEG and Eye Tracking N400 Study. PLoS ONE 10(7): e0134339. doi:10.1371/journal.pone.0134339

Eriksson, M, Westerlund, M., \& Berglund, E. (2002). A screening version of the Swedish communicative development inventories designed for use with 18 months old children. Journal of Speech Language and Hearing Research, 45, 948-960.

Fenson, L., Dale, J. S., Reznick, D., Thal, E., Bates, J., Hartung, S., Pethick, \& Reilly, J. (1993). MacArthur Communicative Inventories: User's Guide and Technical Manual. San Diego: Singular Publishing Group.

Fenson, L., Dale, P. S., Reznick, J. S., Bates, E., Thal, D. J., \& Pethick, S. J. (1994). Variability in early communicative development. Monographs of the Soiety for Research in Child Development, 59, 1-173.

Friedrich, M., \& Friederici, A. D. (2010). Maturing brain mechanisms and developing behavioral language skills. Brain and Language, 114, 66-71.

Gallese, V. (2007) Before and below 'theory of mind': embodied simulation and the neural correlates of social cognition. Philosophical Transactions of the Royal Society A, 362, 659-669.

Gergely, G., Bekkering, H., \& Kiraly, I. (2002). Rational imitation in preverbal infants. Nature, 415, 755. 
Holle, H., \& Gunter, T. C. (2007). The role of iconic gestures in speech disambiguation: ERP evidence. Journal of Cognitive Neuroscience, 19, 11751192.

Hoehl, S., \& Wahl, S. (2012). Recording infant ERP data for cognitive research. Developmental neuropsychology, 37, 187-209.

Hunnius, S. \& Bekkering, H. (2010). The early development of object knowledge: a study of infants' visual anticipations during action observation. Developmental Psychology, 46, 446-54.

Hoorman, J., Falkenstein, M., Schwarzenau, P., \& Hohnsbein, J. (1998). Methods for the quantification and statistical testing of ERP differences across conditions. Behavioral Research Methods: Instruments \& Computers, 31, 103-109.

Kaduk, K., Elsner, B., \& Reid, V. M. (2013). Discrimination of animate and inanimate motion in 9-month-old infants: an ERP study. Developmental cognitive neuroscience, $6,14-22$.

Kelly, S. D., Ward, S., Creigh, P. \& Bartolotti, J. (2007). An intentional stance modulates the integration of gesture and speech during comprehension. Brain and language, 101, 222-233.

Kochukhova, O., \& Gredebäck, G. (2010). Preverbal infants anticipate that food will be brought to the mouth: an eye tracking study of manual feeding and flying spoons. Child Development, 81, 1729-1738.

Kraljević, J., Cepanec, M., \& Šimleša, S. (2014). Gestural development and its relation to child's early vocabulary. Infant Behavior and Development, 37, 192-202.

Kutas, M., \& Hillyard, S. A. (1980). Event-related potentials to semantically inappropriate and surprisingly large words. Biological Psychology, 11, 99- 
116.

Mandler, J. M. (2006). Actions organize the infant's world. In K. Hirsh-Pasek, \& R. Michnick Golinkoff (Eds.), Action Meets Word: How Children Learn Verbs (pp. 111-133). Oxford University press, Inc.

Ni Choisdealbha, A., \& Reid, V. M. (2014). The developmental cognitive neuroscience of action: semantics, motor resonance and social processing. Experimental brain research, 232, 1585-1597.

Parise, E., \& Csibra, G. (2012). Electrophysiological evidence for the understanding of maternal speech by 9-month-old infants. Psychological Science, 23, 728733.

Parise, E., Reid, V. M., Stets, M., \& Striano, T. (2008). Direct eye contact influences the neural processing of objects in 5-month-old infants. Social Neuroscience, $3,141-150$.

Rämä, P., Sirri, L., \& Serres, J. (2013). Development of lexical-semantic language systems: N400 priming effect for spoken words in 18- and 24-month old children. Brain and Language, 125, 1-10.

Reid, V. M., Hoehl, S., Grigutsch, M., Groendahl, A., Parise, E. \& Striano, T. (2009). The neural correlates of infant and adult goal prediction: evidence for semantic processing systems. Developmental Psychology, 45, 620-629.

Reid, V. M. \& Striano, T. (2007). The directed attention model of infant social cognition. European Journal of Developmental Psychology, 4, 100-110.

Reid, V. M., \& Striano, T. (2008). N400 involvement in the processing of action sequences. Neuroscience letters, 433, 93-97.

Rizzolatti, G. \& Arbib, A. A. (1998). Language within our grasp. Trends in Neuroscience, 21, 188-194. 
Reynolds, G. D., \& Richards, J. E. (2005). Familiarization, attention and recognition memory in infancy: An event-related potential and cortical source localization study. Developmental Psychology, 41, 598-615.

Rowe, M. L. \& Goldwin-Meadow, S. (2009a). Early gesture selectively predicts later language learning. Developmental Science, 12, 182-187.

Rowe, M. L. \& Goldwin-Meadow, S. (2009b). Differences in early gesture explains SES disparities in child vocabulary size at school entry. Science, 323, 951953.

Snyder, K., Webb, S.J., \& Nelson, C.A. (2002). Theoretical and methodological implications of variability in infant brain response during a recognition memory paradigm Infant Behavior and Development, 25, 466-494.

Sommerville, J. \& Woodward, A. L. (2005). Pulling out the intentional structure of action: the relation between action processing and action production in infancy. Cognition, 95, 1-30.

Stets, M. \& Reid, V. M. (2011). Infant ERP amplitudes change over the course of an experimental session: Implications for cognitive processes and methodology. Brain and Development, 34, 325-329.

Stets, M., Stahl, D., \& Reid. V. M. (2012). A meta-analysis investigating factors underlying attrition rates in infant ERP studies. Developmental Neuropsychology, 37, 226-252.

Thomas, M. S. C., Annaz, D., Ansari, D., Serif, G., Jarrold, C., \& Karmiloff-Smith, A. (2009). Using developmental trajectories to understand developmental disorders. Journal of Speech, Language, and Hearing Research, 52, 336-358.

Tomasello, M., Carpenter, M., \& Liszkowski, U. (2007). A new look at infant pointing. Child Development, 78, 705-722.

Torkildsen, J., Sannerud, T., Syversen, G., Thormodsen, R., Simonsen, H. G., Moen, 
I., Smith, L., \& Lindgren, M. (2006). Semantic organization of basic-level words in 20-month-olds: An ERP study. Journal of Neurolinguistics, 19, 431454.

Torkildsen, J., Hansen, H. F., Svangstu, J. M., Smith, L., Simonsen, H. G., Moen, I., Lindgren, M. (2008). Brain dynamics of word familiarization in 20-montholds: Effects of productive vocabulary size. Brain and Language, 108, 73-88.

Torkildsen, J., Friis Hansen, H., Scangstu, J. M., Smith, L., Simonsen, H. G., Moen, I., \& Lindgren, M. (2009). Brain dynamics of word familiarization in 20month-olds: effects of productive vocabulary size. Brain and language, 108, 73-88.

Zacks, J. M., Braver, T. S., Sheridan, M. A., Donaldson, D. I., Snyder, A. Z., Ollinger, J. M., Buckner, R. L., \& Raichle, M. E. (2001). Human brain activity time-locked to perceptual event boundaries. Nature Neuroscience, 4, 651-655.

Webb, S. J., Long, J. D., \& Nelson, C. A. (2005). A longitudinal investigation of visual event-related potentials in the first year of life. Developmental Science, 8, 605-616.

Wu, Y. C., \& Coulson, S. (2005). Meaningful gestures: Electrophysiological indices of iconic gesture comprehension. Psychophysiology, 42, 654 - 667. 


\section{Highlights}

- Infants at 9 months were tested for producing an N400 for action semantics

- Only those infants who had higher than mean language comprehension at 9 months and language production skills at 18 months produced an N400 at 9 months

- Infants with a lower than mean language comprehension and production capacity did not evidence an N400 at 9 months of age

- Similarities in action and language processing indicate shared sequence processing across these cognitive domains 
Click here to download high resolution image
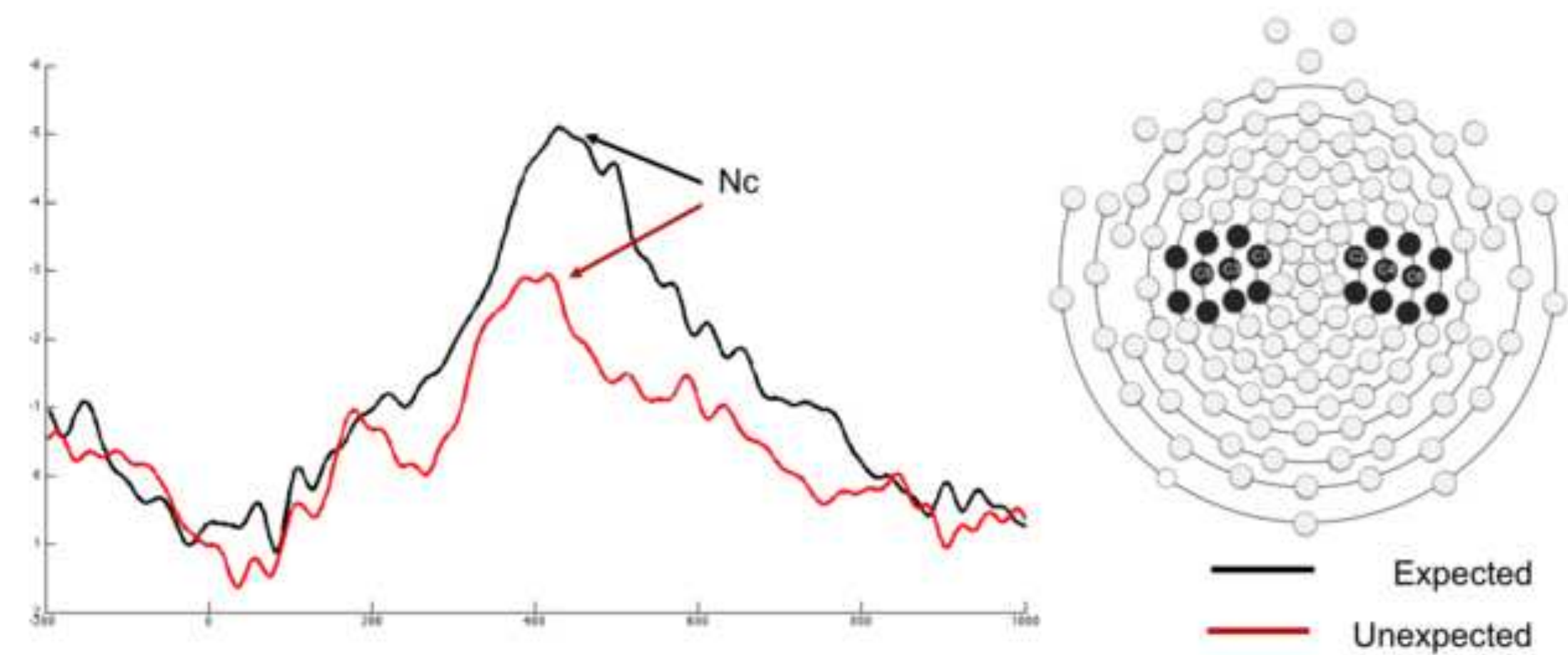

here to download high resolution image

Unexpected 

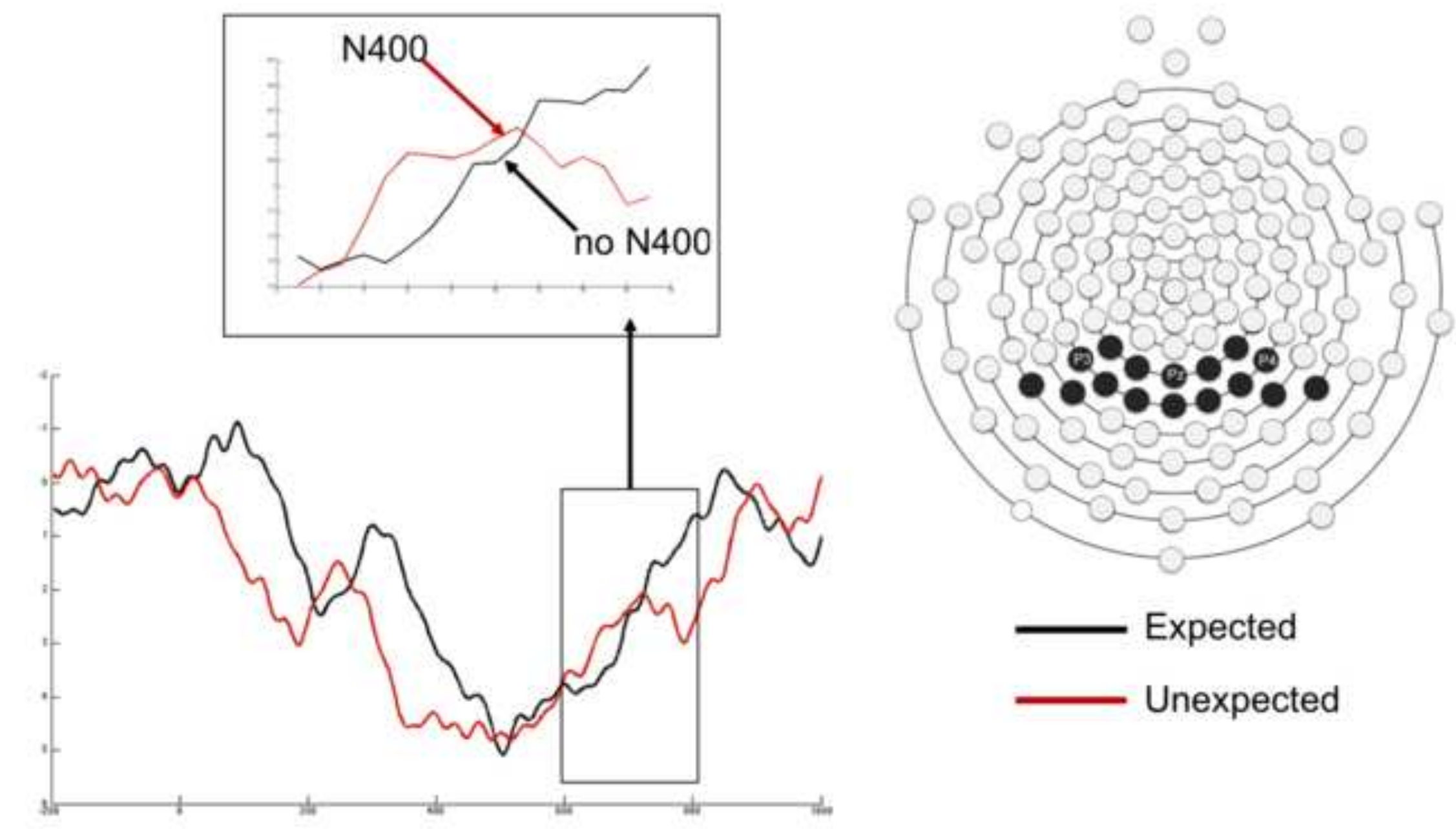

Unexpected 
Click here to download high resolution image

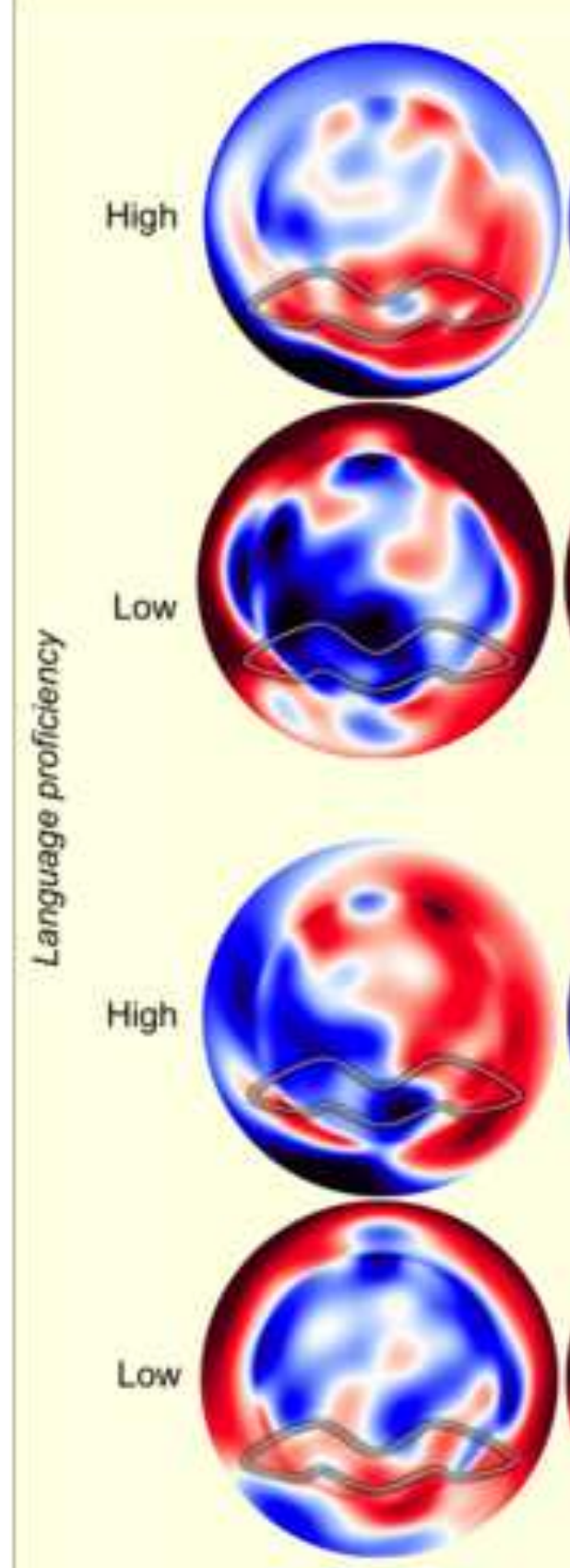

600

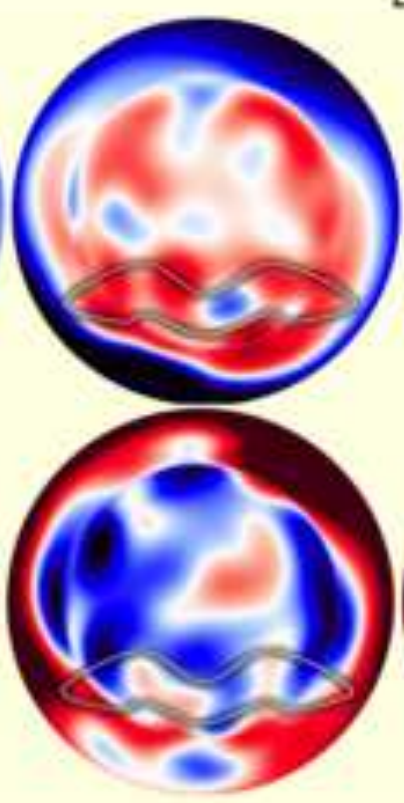

Language Comprehension
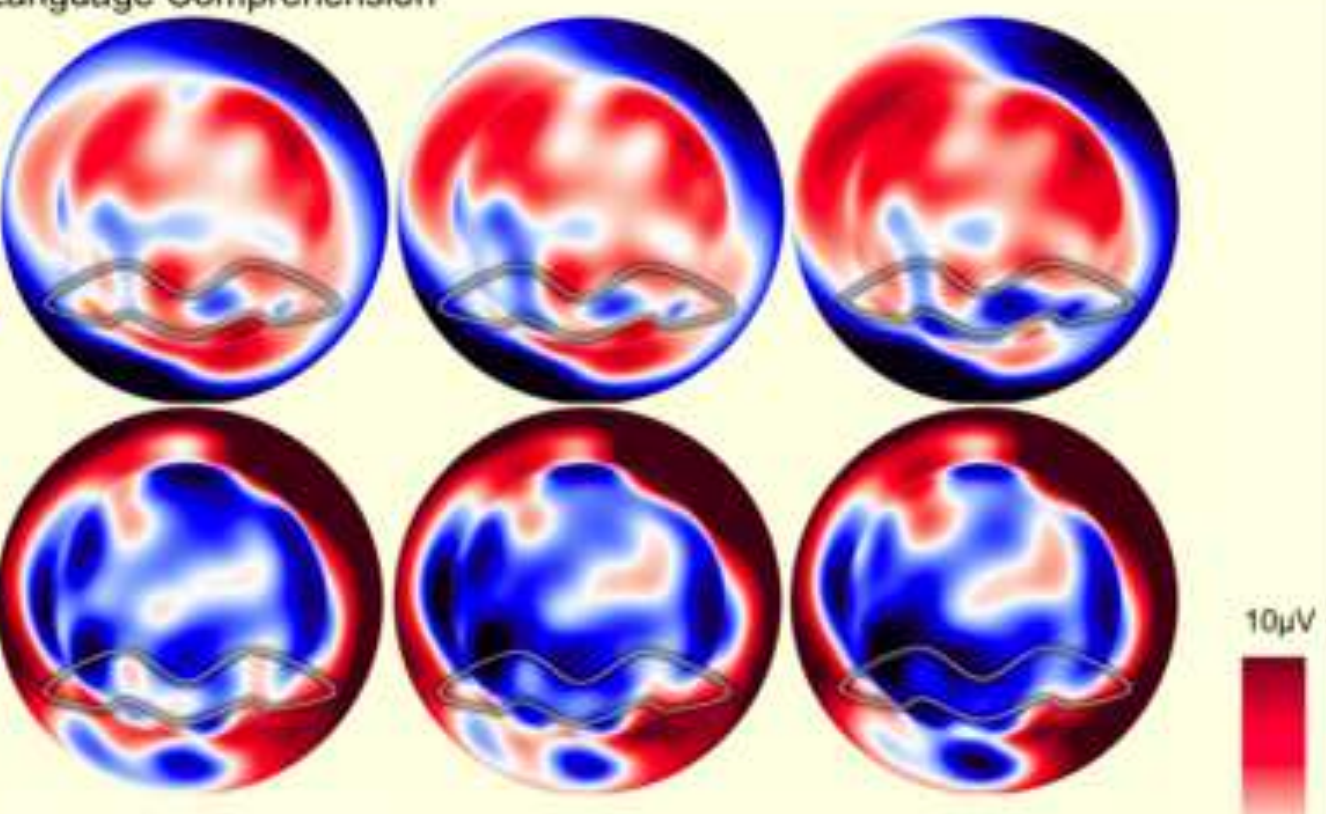

Language Production
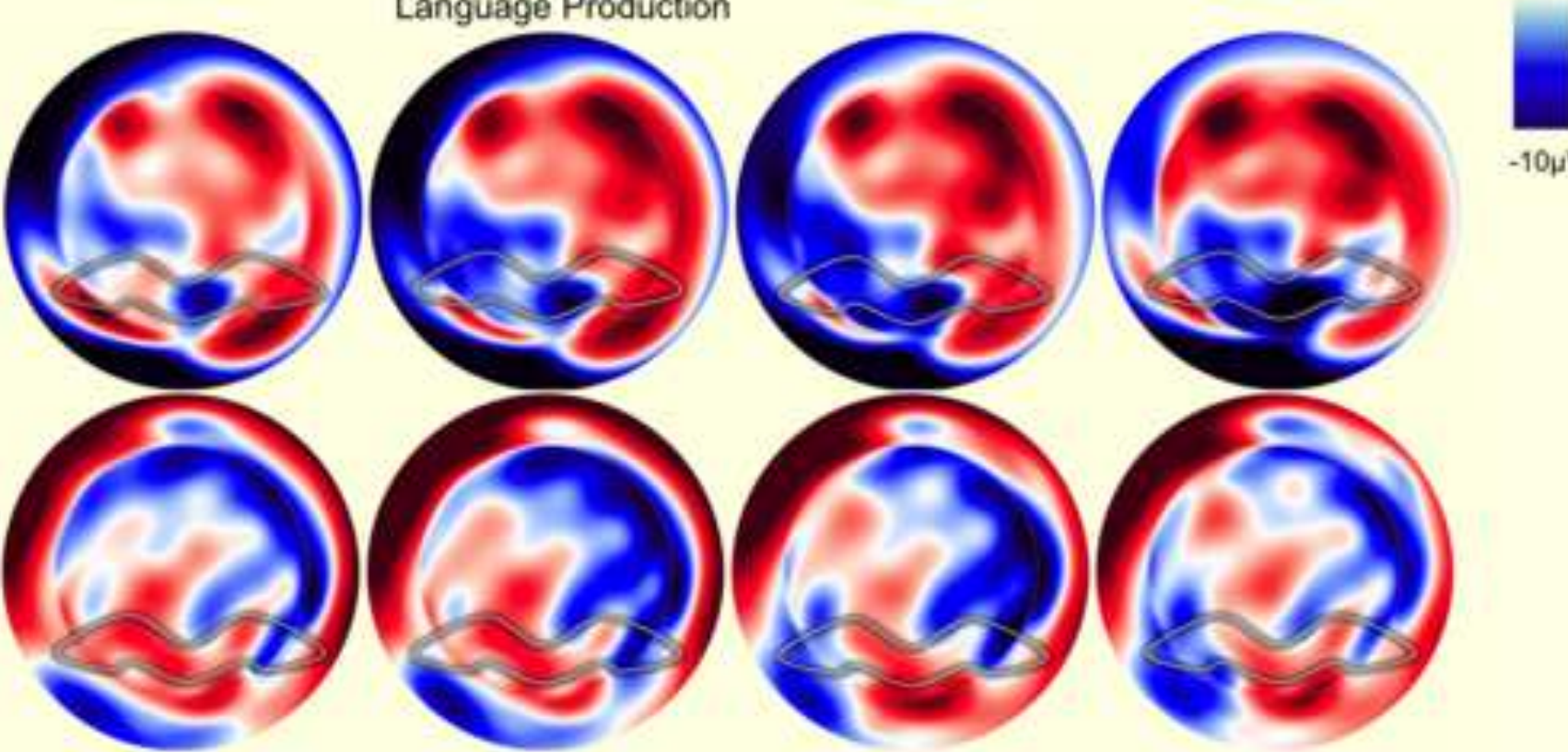

$-10 \mu \mathrm{V}$

700

750

800

Time in milliseconds 